\title{
Efecto de la concentración de nitrógeno sobre atributos morfológicos, potencial de crecimiento radical y estatus nutricional en plantas de Eucalyptus globulus producidas a raíz cubierta
}

\author{
Effect of the nitrogen concentration on the morphological attributes, root growth potential \\ and nutritional status on cover root Eucalyptus globulus seedlings \\ Jordán Monsalvea, René Escobara, Manuel Acevedoa*, Manuel Sánchez ${ }^{\mathrm{a}}$, Rafael Coopmana, b \\ *Autor de correspondencia: aniversidad de Concepción, Facultad de Ciencias Forestales, Victoria 631, \\ casilla 160-C, Concepción, Chile, maacevedo@udec.cl \\ bUniversidad Austral de Chile, Facultad de Ciencias Forestales, Valdivia, Chile.
}

\begin{abstract}
SUMMARY
Four nitrogen concentrations $\left(50,100,150\right.$, and $\left.200 \mathrm{mg} \mathrm{L}^{-1}\right)$ were evaluated on shoot and root growth, root growth potential, and nutritional status of cover root Eucalyptus globulus seedlings. Nutrient solutions were applied on a weekly basis together with irrigation. All other elements were held constant in the nutrient solutions. Irrigation was applied considering a $20 \%$ loss of the seedling tray. Plant height and leaf area reached their peak at $200 \mathrm{mg} \mathrm{L}^{-1}$; collar diameter, number and the absolute weight of new roots did not change when concentrations where 150 or $200 \mathrm{mg} \mathrm{L}^{-1}$. For all treatments the best results were obtained when the fertilization was made with $200 \mathrm{mg} \mathrm{L}^{-1}$ because it produced plants with higher root growth potential and better morphological attributes, with the only disadvantage of low levels of foliar nitrogen obtained at the end of the essay.
\end{abstract}

Key words: Eucalyptus globulus, fertilization with nitrogen, nutritional state, morphological attributes.

\section{RESUMEN}

Se evaluó el efecto de la aplicación de cuatro concentraciones de nitrógeno (50, 100, 150 y $200 \mathrm{mg} \mathrm{L}^{-1}$ ) sobre atributos morfológicos, potencial de crecimiento radical y estatus nutricional de plantas de Eucalyptus globulus producidas a raíz cubierta. La frecuencia de aplicación fue semanal, manteniendo constantes los niveles de los otros macroelementos. Además, se consideró un esquema de riego al $20 \%$ de disminución de pérdida de peso de bandeja. Las variables altura de planta y área foliar alcanzaron su máximo valor al fertilizar con $200 \mathrm{mg} \mathrm{L}^{-1}$, mientras que para el diámetro de cuello y número de raíces nuevas los valores obtenidos no difieren estadísticamente al fertilizar con concentraciones de 150 y $200 \mathrm{mg} \mathrm{L}^{-1}$. Para el rango de concentraciones ensayadas se concluye que los mejores resultados se obtienen al fertilizar con $200 \mathrm{mg} \mathrm{L}^{-1}$, ya que produce plantas con un mayor potencial de crecimiento radical y mejores atributos morfológicos, siendo la única desventaja los bajos niveles de nitrógeno foliar obtenidos al finalizar el ensayo.

Palabras clave: Eucalyptus globulus, fertilización nitrogenada, estatus nutricional, atributos morfológicos.

\section{INTRODUCCIÓN}

El éxito de una plantación forestal depende de la calidad de las plantas, la que está definida por su comportamiento final en terreno. Este último está regulado por los atributos morfológicos y fisiológicos de las plantas y por su interacción con el ambiente en el sitio de plantación (Duryea y McClain 1984). Las prácticas de vivero ayudan a mejorar el desempeño de las plantas en terreno (Landis 1985, Howell y Harrington 2004). Sin embargo, los atributos morfofuncionales y las prácticas culturales para obtenerlos están todavía bajo discusión (Cortina et al. 2006). Para el caso de Eucalyptus spp., las prácticas utilizadas tradicionalmente en vivero incluyen, entre otras, manejo radical (poda de raíces y descalces), manejo de tallo (poda de ramas laterales y apical), manejo del riego y fertilización, que en conjunto influyen en el endurecimiento de la planta, el potencial de crecimiento radical (PCR) y su incremento en la resistencia a las heladas (Arévalo 1994).

La fertilización es una de las prácticas culturales más importantes para la obtención de plantas de buena calidad para la forestación, especialmente de aquella producida en contenedores donde el volumen limitado de sustrato dificulta seriamente su crecimiento (Landis 2000). La fertilización puede acelerar el crecimiento aéreo y radical de las plantas, modifica el contenido de nutrientes para luego cambiar la cantidad de reservas disponibles, mejorando el enraizamiento en terreno y el crecimiento, la resistencia al estrés hídrico, a bajas temperaturas y enfermedades 
(Landis 1985, Van den Driessche 1991, 1992, Timmer y Aidelbaum 1996, Haase y Rose 1997, Shaw et al. 1998, Malik y Timmer 1998, Grossnickle 2000). Según diversos autores (Navarro y Pemán 1997, Peñuelas y Ocaña 2000), la concentración de cada nutriente en la solución del medio de cultivo es el aspecto más importante en un programa de fertilización. Una concentración demasiado baja reduce el crecimiento, mientras que concentraciones altas producen excesos de salinidad y afectan el crecimiento y calidad de las plantas.

Otro aspecto importante en un programa de fertilización es la frecuencia de aplicación. Al respecto, Close et al. (2005) evaluaron el efecto de la frecuencia de aplicación de fertilizantes en base de nitrógeno $(1,25 \mathrm{mg}$ por planta de Peters Excel, 20:2, 2:6,6), en plantas de Eucalyptus globulus Labill. y Eucalyptus nitens (Deane et Maiden) Maiden, tanto en vivero como en plantación, encontrando niveles de deficiencias de nitrógeno (\% de materia seca de hojas), tanto para una como para dos aplicaciones semanales, durante su permanencia en vivero. Por otra parte, Materán et al. (2004) evaluaron el efecto de la aplicación de cinco esquemas de fertilización nitrogenada sobre el desarrollo de plantas de E. globulus producidas en contenedores. Concluyeron que la fertilización nitrogenada aumenta significativamente la biomasa aérea y radical, el crecimiento en diámetro de cuello y altura de plantas, obteniéndose los mejores resultados con el medio nutritivo Driver Kunikuyi (DKW), con 27,8 mg por planta de nitrato de amonio.

La necesidad de caracterizar el efecto que poseen las labores culturales en vivero, sobre los atributos morfológicos, fisiológicos y del comportamiento de las plantas producidas, y la posterior retroalimentación que debe generarse respecto del desempeño que el cultivos manifiesta en terreno, son elementos claves en la repoblación de masas boscosas.

Por las razones expuestas, el objetivo de este estudio es evaluar el efecto de la aplicación de cinco concentraciones de nitrógeno sobre el desarrollo en vivero de plantas de $E$. globulus producidas en contenedores. En forma particular, se pretende evaluar el efecto de las concentraciones de nitrógeno sobre los principales atributos morfológicos y el potencial de crecimiento radical. Evaluar el efecto de estas concentraciones aplicadas sobre el estatus nutricional de las plantas al final del ensayo y determinar si la fertilización nitrogenada permite controlar el crecimiento en altura de las plantas durante la fase de pleno crecimiento.

\section{MÉTODOS}

El ensayo fue establecido en el vivero forestal Quinchamalí, perteneciente a la Sociedad Agrícola y Forestal Proplantas Limitada, ubicado en el kilómetro 22 del camino que une Chillán con Quinchamalí en la Región del Biobío $\left(36^{\circ} 43^{\prime} \mathrm{S}, 72^{\circ} 06^{\prime} \mathrm{O}\right)$. La zona presenta un clima medi- terráneo templado, que se caracteriza por una temperatura media anual de $14^{\circ} \mathrm{C}$, con una máxima media del mes más cálido (enero) de $28,8^{\circ} \mathrm{C}$ y una mínima media del mes más frío (julio) de $3,5^{\circ} \mathrm{C}$. El período libre de heladas es de noviembre a marzo; el régimen hídrico se caracteriza por una precipitación anual de $1.025 \mathrm{~mm}$, siendo el mes de julio el más lluvioso, con $217 \mathrm{~mm}$, y un mínimo en enero con $16 \mathrm{~mm}$ (Novoa y Villaseca 1989).

El material vegetal utilizado para la evaluación del ensayo correspondió a plantas provenientes de semillas proporcionadas por el vivero desde su producción comercial. El comienzo del ensayo se efectuó cuando las plantas alcanzaron el completo establecimiento del sistema radical en los contenedores, el cual fue evaluado en forma visual. La aplicación de las dosis de nitrógeno tuvo una duración de 10 semanas, desde marzo a mayo de 2005, que abarcó la fase de pleno crecimiento. La posterior evaluación de los atributos morfológicos y del potencial de crecimiento radical de las plantas producidas se realizó en el Laboratorio de Fisiología de Árboles de la Facultad de Ciencias Forestales de la Universidad de Concepción, en Concepción, al término del proceso de producción.

Se utilizaron 32 bandejas de poliestireno expandido de 112 cavidades con $100 \mathrm{~cm}^{3}$ de sustrato. Las cavidades en su interior fueron bañadas con una solución con cobre a objeto de obtener la poda química de raíces, según la mezcla utilizada por el vivero para esta labor. El sustrato utilizado fue compost de corteza de pino (Pinus radiata D. Don) con una granulometría de $74,3 \%$ menor a $3 \mathrm{~mm}$; $20,7 \%$ entre 3 y $5 \mathrm{~mm}$ y $5,0 \%$ mayor a $5 \mathrm{~mm}$. Así, en el medio de crecimiento se originó una porosidad de retención $26,9 \% ; 25,1 \%$ en aireación y un $52 \%$ porosidad total.

La fertilización se basó en la aplicación de dosis crecientes de nitrógeno, las cuales correspondieron a 50, 100,150 y $200 \mathrm{mg} \mathrm{L}^{-1}$, manteniendo constante el resto de los macroelementos en $60 \mathrm{mg} \mathrm{L}^{-1}$ para el fósforo, $100 \mathrm{mg} \mathrm{L}^{-1}$ para el potasio, $80 \mathrm{mg} \mathrm{L}^{-1}$ para el calcio, $40 \mathrm{mg} \mathrm{L}^{-1}$ para el magnesio y $60 \mathrm{mg} \mathrm{L}^{-1}$ para el azufre. Las fuentes utilizadas para los distintos macroelementos fueron las siguientes sales: urea $\left(\mathrm{CO}\left(\mathrm{NH}_{2}\right)_{2}\right)$, nitrato de amonio $\left(\mathrm{NH}_{4} \mathrm{NO}_{3}\right)$, nitrato de calcio $\left(\mathrm{Ca}\left(\mathrm{NO}_{3}\right)_{2}\right)$, nitrato de potasio $\left(\mathrm{KNO}_{3}\right)$, fosfato monoamónico $\left(\mathrm{NH}_{2} \mathrm{H}_{2} \mathrm{PO}_{4}\right)$, fosfato monopotásico $\left(\mathrm{KH}_{2} \mathrm{PO}_{4}\right)$, cloruro de potasio $(\mathrm{KCl})$ y sulfato de magnesio $\left(\mathrm{MgSO}_{4}\right)$.

Se realizó el análisis químico del agua de riego del vivero a objeto de determinar el aporte de elementos nutritivos por parte de ésta, incorporando este antecedente al balance de las soluciones generadas. Además, se verificó el pH del agua de riego, no siendo necesaria su modificación de acuerdo al valor objetivo cifrado de 6,5. La preparación de las soluciones se realizó siguiendo la metodología descrita por Landis (2000). Las soluciones fueron preparadas en forma concentrada, considerando para ello la solubilidad de las sales utilizadas. Al momento de la fertilización las soluciones se diluyeron en agua, hasta alcanzar los 50 
litros. La fertilización se llevó a cabo mediante la inmersión de las bandejas en tinas, obteniendo una fertilización a saturación por capilaridad. Los fertilizantes se aplicaron dos veces por semana, siempre al momento de alcanzar el criterio de riego. La fertilización de cada bandeja se realizó en dos etapas, primero la inmersión de la bandeja en la solución con nitrato de calcio $\mathrm{Ca}\left(\mathrm{NO}_{3}\right)_{2}$ y luego en otra tina que contenía la solución con los demás elementos, esto para evitar la precipitación del calcio con los sulfatos y fosfatos utilizados.

El manejo del riego fue controlado por el monitoreo de la pérdida del peso de la bandeja, el cual fue medido dos veces al día. El criterio para regar fue la pérdida del $20 \%$ del peso de la bandeja. Una vez alcanzado este nivel, las bandejas fueron regadas mediante el método de inmersión dentro de un recipiente con agua, obteniendo un riego a saturación por capilaridad. Cada 10 días, se calibró el agua aprovechable de las bandejas, producto del aumento en biomasa vegetal al peso de bandeja.

Para la evaluación del material, se dejó una zona de borde correspondiente a las bandejas que estaban en el contorno del mesón de producción. Además, no se evaluaron las plantas periféricas dentro de cada bandeja, con el fin de evitar el efecto borde y plantas con algún tipo de daño producto de la manipulación de las bandejas.

La variable altura total $(\mathrm{cm})$ fue medida semanalmente durante todo el ensayo. Para ello se seleccionaron y marcaron cinco plantas por bandeja al inicio de éste. La medición se realizó desde el cuello hasta el ápice, con una huincha de medir con precisión de $1 \mathrm{~mm}$. Los otros atributos morfológicos se midieron al término del ensayo, los cuales correspondieron a:

- Área foliar $\left(\mathrm{cm}^{2}\right)$ : con un planímetro electrónico LICOR 3100 , obteniendo el promedio de tres mediciones consecutivas del área foliar, con precisión de $0,05 \mathrm{~cm}^{2}$.

- Diámetro altura del cuello (mm): para esta medición se ocupó un pie de metro digital Mitutoyo con precisión de $0,01 \mathrm{~mm}$.

Para evaluar la capacidad del sistema radical de formar raíces nuevas se estableció un ensayo de potencial de crecimiento radical (PCR) con 20 plantas por tratamiento. Una vez colectadas aleatoriamente las muestras, se lavaron los sistemas radicales y se recortaron, extrayendo las raíces nuevas no suberizadas presentes, a objeto de que todas las plantas pertenecientes a cada uno de los tratamientos comenzaran el ensayo sin raíces nuevas ya formadas. Luego, el ensayo fue montado en una cámara aeropónica, la cual con una frecuencia de riego de 10 minutos inyecta agua sin elementos nutritivos hacia el sistema radical durante seis segundos; se mantuvo la temperatura de la llovizna a $22^{\circ} \mathrm{C}$ y el fotoperíodo fue de 16 horas, proporcionado por tubos fluorescentes instalados sobre la cámara, los cuales produjeron un flujo fotónico promedio a nivel de follaje de $85 \mu \mathrm{mol} \mathrm{m}{ }^{-2} \mathrm{~s}^{-1}$. El ensayo duró un período de 28 días. La evaluación del ensayo se realizó al finalizar este período considerando las siguientes variables de medición:

- Número de raíces nuevas $\left(\mathrm{N}^{\circ} \mathrm{RN}\right)$ : a través del conteo de las raíces no suberizadas $>1 \mathrm{~cm}$ de largo, que se caracterizan por su color blanco.

- Longitud de raíces nuevas (LRN): consistió en medir, con precisión de $0,1 \mathrm{~cm}$, la longitud de las tres raíces nuevas más largas, de longitud $\geq 1 \mathrm{~cm}$, para posteriormente calcular el promedio de las longitudes.

- Biomasa de raíces nuevas (BRN): consistió en secar la totalidad de raíces nuevas por $48 \mathrm{~h}$ a $85^{\circ} \mathrm{C}$ en un horno de ventilación forzada, para su posterior pesaje en una balanza digital de precisión de 0,001 g.

Para los análisis químicos, se extrajeron dos muestras aleatorias de cinco plantas por tratamiento, a las cuales se les determinaron los niveles de nitrógeno, fósforo, potasio, calcio y magnesio. Se siguieron los métodos de nitrógeno total mediante digestión Kjeldahl y determinación mediante método colorimétrico; para el fósforo mediante calcinación por vía seca, disolución en ácido clorhídrico $1 \mathrm{~N}$ y determinación por método colorimétrico; en el caso del potasio mediante calcinación por vía seca, disolución con ácido clorhídrico $1 \mathrm{~N}$ y determinación por espectrofotometría de emisión atómica; finalmente, para el calcio y el magnesio mediante calcinación por vía seca, disolución en ácido clorhídrico $1 \mathrm{~N}$ y determinación por espectrofotometría de absorción atómica.

El diseño experimental utilizado correspondió al de bloques completos al azar (Little y Hills 1978), con cuatro tratamientos correspondientes a la concentración de nitrógeno. La unidad experimental estuvo conformada por una bandeja de 112 plantas, con ocho réplicas para cada tratamiento. Los resultados fueron analizados estadísticamente, comprobando su independencia, normalidad y homogeneidad de varianzas. Las diferencias entre medias fueron contrastadas con el método de comparación múltiple de Tuckey (Montgomery 1991), mediante el software analítico Statistica versión 6.0.

\section{RESULTADOS}

Se observó un aumento significativo en la altura promedio de las plantas a partir de la quinta semana del ensayo, aumentando estas diferencias en el tiempo hasta llegar al final del ensayo (figura 1). A medida que aumentó la concentración de nitrógeno, también lo hizo el crecimiento en altura, llegando a obtenerse en promedio alturas totales de 32,$5 ; 35,6 ; 39,8$ y $44,5 \mathrm{~cm}$ para los tratamientos de 50 , 100,150 y $200 \mathrm{mg} \mathrm{L}^{-1}$, respectivamente.

Al final del ensayo se observó para el diámetro a la altura del cuello (DAC) un aumento significativo desde el tratamiento de 50 hacia el de $200 \mathrm{mg} \mathrm{L}^{-1}$, con incrementos decrecientes a medida que aumentó la concentración de 


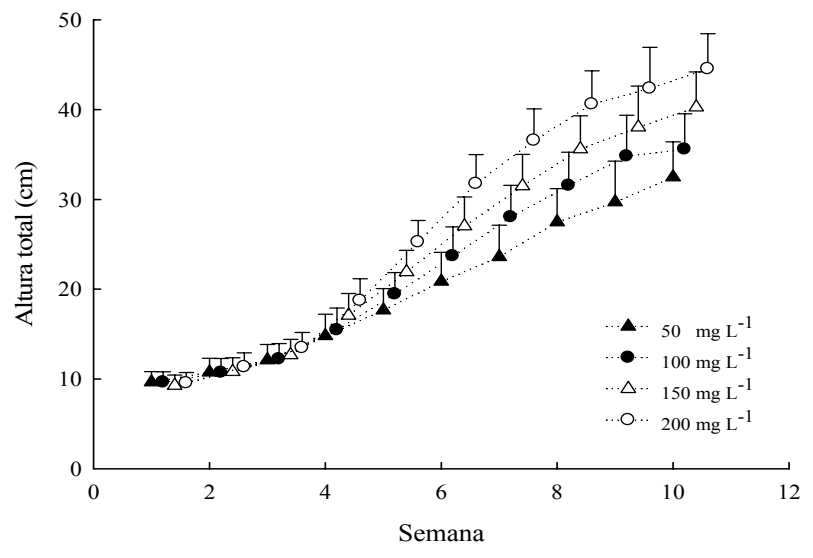

Figura 1. Efecto de la concentración de nitrógeno sobre el crecimiento en altura en plantas de E. globulus cultivadas en contenedores (no traslape del intervalo, según Tuckey, indica diferencia significativa, $P \leq 0,05)$.

Effect of nitrogen concentration over the average height on E. globulus plants cultivated on containers (non overlap of the interval, according to Tuckey, differ significantly, $P \leq 0,05$ ).

este elemento (figura 2A). Las tres concentraciones más altas de nitrógeno sólo generaron diferencias significativas entre los tratamientos de 100 y $200 \mathrm{mg} \mathrm{L}^{-1}$.

Se observó un aumento significativo del área foliar a medida que aumentó la concentración de nitrógeno en el medio de crecimiento (figura 2B). Con la concentración de $200 \mathrm{mg} \mathrm{L}^{-1}$ de nitrógeno se produjo un considerable aumento del área foliar, alcanzando un valor máximo promedio de $196 \mathrm{~cm}^{2}$.

Respecto del potencial de crecimiento radical (figura 3), para las variables número de raíces nuevas y biomasa de raíces nuevas, se observó un aumento en la respuesta a medida que aumentó la concentración de nitrógeno en el medio de crecimiento, siendo el efecto de los tratamientos de 200 y $150 \mathrm{mg} \mathrm{L}^{-1}$ de nitrógeno iguales entre sí, pero estadísticamente superiores a las respuestas obtenidas con las concentraciones de 100 y $50 \mathrm{mg} \mathrm{L}^{-1}$. Para la variable longitud de raíces nuevas, no se obtuvieron diferencias significativas entre las concentraciones de nitrógeno suministradas.

Los análisis foliares realizados al final del ensayo (cuadro 1) mostraron un aumento en los niveles de nitrógeno foliar con el aumento de la concentración de la fertilización nitrogenada. No se observó ninguna tendencia para los niveles foliares del resto de los elementos como consecuencia de relaciones de antagonismo o sinergismo que se producen a nivel de medio de crecimiento que posteriormente pueden ser detectados a nivel foliar.

\section{DISCUSIÓN}

Los resultados observados para la altura promedio coinciden con lo planteado por Landis (2000), quien señala
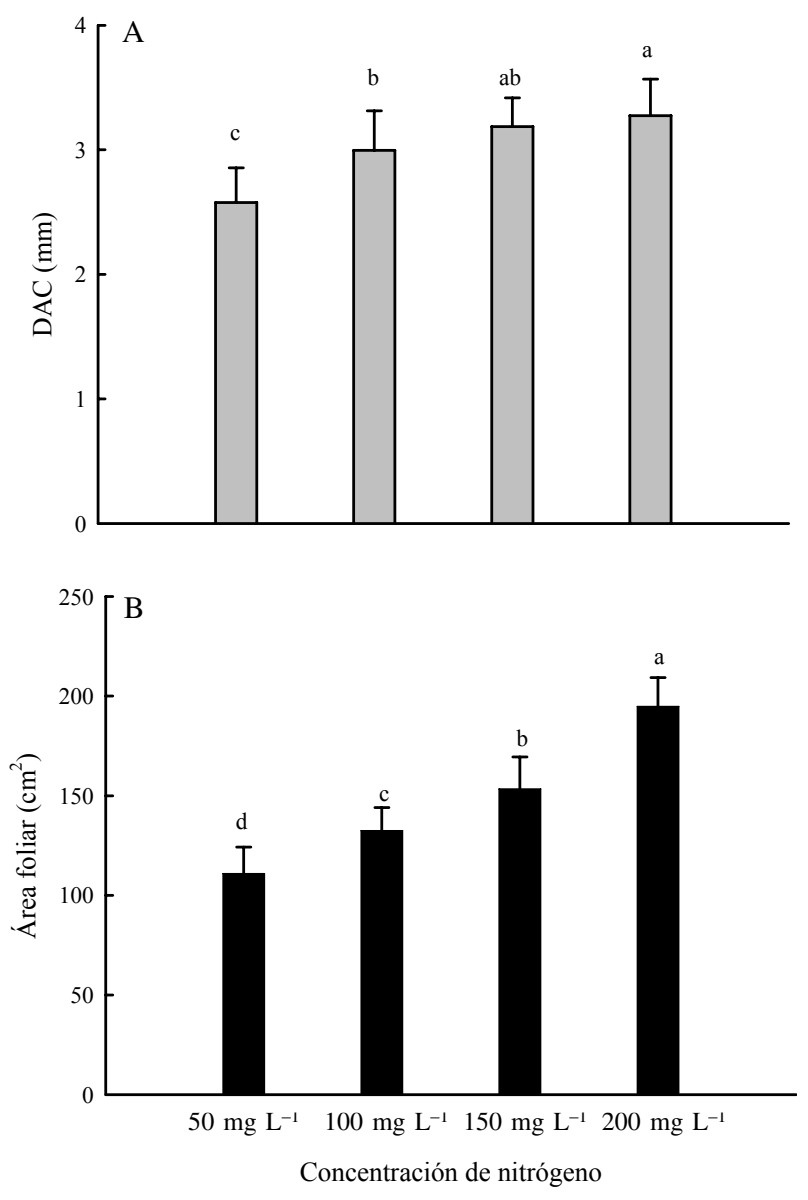

Figura 2. Efecto de la concentración de nitrógeno en plantas de E. globulus cultivadas en contenedores: A) crecimiento diametral y B) el área foliar. (Barras indican \pm desviación estándar. Diferentes letras, según Tuckey, difieren significativamente, $P \leq 0,05$.)

Effect of nitrogen concentration over E. globulus plants cultivated on containers. A) diametral growth and B) leaf area (Bars indicate \pm standard deviation. Different letters, according to Tuckey, differ significantly, $P \leq 0,05)$.

que niveles elevados de nitrógeno promueven una rápida división y elongación celular. En tanto, Salisbury y Ross (2000) plantean que el nitrógeno se encuentra presente en muchos compuestos esenciales, no sorprendiendo en absoluto que el crecimiento sea lento cuando existe una baja disponibilidad de nitrógeno. Estos resultados confirman los conceptos reportados por algunos autores en cuanto a la tendencia al incremento en la altura de las plantas al aplicarse una fertilización nitrogenada junto con otros elementos, como el fósforo y potasio (Wang et al. 1988, Materán et al. 2004, Close et al. 2005).

Hasta la segunda medición no se observan diferencias significativas en altura, debido a que se efectuó sólo una fertilización y las respuestas en crecimiento producto de la fertilización nunca son inmediatas. A partir de la tercera semana, hay una clara diferencia que marca el tratamiento de $200 \mathrm{mg} \mathrm{L}^{-1}$ sobre los tratamientos de 50 

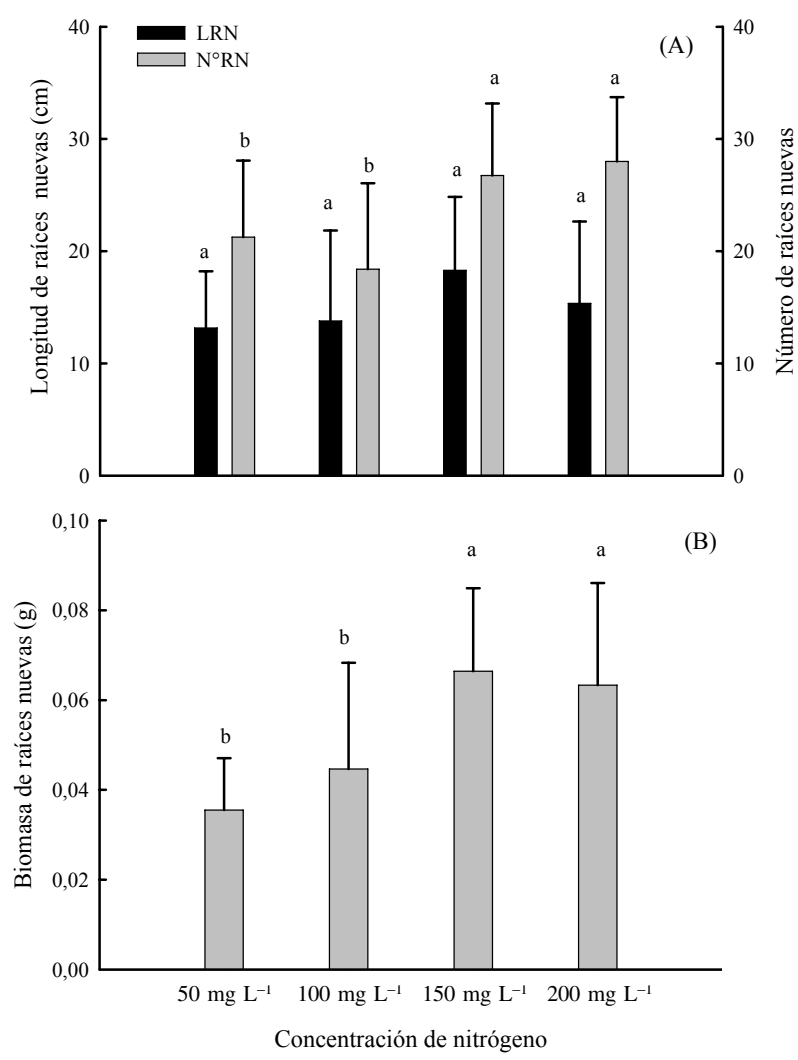

Figura 3. Efecto de la dosis de nitrógeno sobre el potencial de crecimiento de la raíz en plantas de E. globulus cultivadas en contenedores: A) longitud de raíces nuevas (LRN) producidas y número de raíces nuevas $\left(\mathrm{N}^{\circ} \mathrm{RN}\right)$ producidas; y $\left.\mathrm{B}\right)$ biomasa de las raíces producidas. (Barras indican \pm desviación estándar. Diferentes letras, según Tuckey, difieren significativamente, $P \leq 0,05)$.

Effect of nitrogen doses over root growth potential on $E$. globulus plants cultivated on containers. A) New root length produced and number of new root produced and B) Biomass of produced root area (Bars indicate \pm standard deviation. Different letters, according to Tuckey, differ significantly, $P \leq 0,05)$.

y $100 \mathrm{mg} \mathrm{L}^{-1}$. Este resultado concuerda con la respuesta a la fertilización obtenida por Materán et al. (2004). En la cuarta semana, se encontraron diferencias significativas entre los tratamientos de 200 y $150 \mathrm{mg} \mathrm{L}^{-1}$, pero aún no se visualizaron diferencias entre los tratamientos de $50 \mathrm{y}$ $100 \mathrm{mg} \mathrm{L}^{-1}$. En la quinta semana se observan diferencias significativas entre los cuatro tratamientos, cuya tendencia va en aumento, lo que se traduce en que al cabo de seis aplicaciones de fertilizantes se observa una clara diferencia en la altura de plantas. La implicancia práctica de este resultado radica en que, por ejemplo, si se fijara una altura promedio final de $30 \mathrm{~cm}$ para el cultivo de plantas en esta etapa del cultivo, con un esquema de fertilización $200 \mathrm{mg} \mathrm{L}^{-1}$, se alcanzaría este resultado en 40 días; con el tratamiento de $150 \mathrm{mg} \mathrm{L}^{-1}$ se lograrían una semana más tarde (47 días); con el tratamiento de $100 \mathrm{mg} \mathrm{L}^{-1}$ se tardarían 53 días de cultivo; $y$, finalmente, con el tratamiento de $50 \mathrm{mg} \mathrm{L}^{-1}$ se obtendría esa altura en 63 días. Por ello, la fertilización nitrogenada sería una valiosa herramienta de manejo en lo que respecta a altura de plantas, ya que se podría aumentar o disminuir el tiempo de cultivo. Esta reducción en el tiempo de cultivo implica una serie de ahorros en insumos, agua, electricidad, mano de obra y supervisiones, entre otros.

Para el diámetro de cuello, la disminución de la magnitud en la respuesta de esta variable con el aumento de la concentración de nitrógeno puede ser explicada porque el factor densidad de cultivo comienza a limitar la respuesta para esta variable y no por el hecho de que este elemento no tenga efecto a esas concentraciones.

Respecto del área foliar, el aumento significativo de esta variable se produce a igual número de hojas por plantas entre los distintos tratamientos, por lo que este resultado está determinado sólo por hojas más grandes. Esto genera consecuencias en las plantas, ya que las que presentan mayor área foliar generan marchitez a niveles mucho menores de pérdida de agua desde el contenedor. Obviamente, esto debiese estar ligado a un mayor número de estomas por hoja o a estomas mucho más grandes. Por lo tanto, el viverista, al momento de seleccionar un esquema de fertilización nitrogenada, debe considerar un criterio de riego apropiado, que le permita generar un crecimiento sostenido y equilibrado durante la etapa de pleno crecimiento del cultivo. Este efecto adquiere mayor importancia si se pretende endurecer plantas posteriormente mediante ciclos de disminución del agua disponible en el sustrato.

Para la prueba del potencial de crecimiento radical, todas las respuestas de los tratamientos pertenecen a la categoría 4 de la escala semicuantitativa de potencial de crecimiento radical propuesta por Burdett (1979). Esto significa que la planta, en promedio, genera entre $11 \mathrm{y}$ 30 nuevas raíces mayores a un centímetro de largo. Los resultados obtenidos para este atributo concuerdan con lo reportado por Fernández et al. (2007), quienes probaron el efecto de esquemas de fertilización con distintos niveles de N-P-K en plantas de E. globulus producidas en contenedores, sobre el potencial de crecimiento radical de las plantas al final de ciclo de producción y la posterior sobrevivencia en terreno. Estos autores encontraron que los tratamientos de alta $\left(126 \mathrm{mg} \mathrm{L}^{-1}\right)$ y baja $\left(12,6 \mathrm{mg} \mathrm{L}^{-1}\right)$ concentración de nitrógeno lograron inducir niveles foliares de 1,58 y $0,89 \%$, respectivamente. Dichos niveles mostraron efectos significativos sobre la producción de raíces nuevas y, posteriormente, la sobrevivencia en terreno de estas plantas (un año después de realizada la plantación) fue de 85 y $40 \%$ para los tratamientos de alta y baja concentración, respectivamente.

Los niveles de macronutrientes foliares muestran que con los tratamientos 150 y $200 \mathrm{mg} \mathrm{L}^{-1}$ se obtuvieron los mayores porcentajes de nitrógeno a nivel foliar. Sin embargo, estos niveles se encuentran bajo el rango recomendado como óptimo, por lo que las plantas se encontrarían en un intervalo de deficiencia llamado "necesidad oculta" según lo propuesto por Landis (2000), ya que no se observa- 
Cuadro 1. Efecto de la dosis de nitrógeno en los macronutrientes foliares en plantas de E. globulus cultivadas en contenedores (valores promedio \pm desviación estándar).

Effect of nitrogen dose on foliar macronutrients in E. globulus plants cultivated on containers (mean values \pm standard deviation).

\begin{tabular}{cccccc}
\hline & \multicolumn{5}{c}{ Niveles foliares obtenidos para los tratamientos (\%) } \\
\hline Elemento & $50 \mathrm{mg} \mathrm{L}^{-1}$ & $100 \mathrm{mg} \mathrm{L}^{-1}$ & $150 \mathrm{mg} \mathrm{L}^{-1}$ & $200 \mathrm{mg} \mathrm{L}^{-1}$ & Niveles óptimos* \\
\hline $\mathrm{N}$ & $1,17 \pm 0,03$ & $1,36 \pm 0,06$ & $1,44 \pm 0,01$ & $1,64 \pm 0,04$ & $1,7-3,0$ \\
$\mathrm{P}$ & $0,15 \pm 0,01$ & $0,16 \pm 0,00$ & $0,16 \pm 0,00$ & $0,18 \pm 0,01$ & $0,16-0,26$ \\
$\mathrm{~K}$ & $1,28 \pm 0,02$ & $1,16 \pm 0,01$ & $1,19 \pm 0,04$ & $1,13 \pm 0,03$ & $0,7-1,5$ \\
$\mathrm{Ca}$ & $1,02 \pm 0,05$ & $0,70 \pm 0,03$ & $1,05 \pm 0,00$ & $1,10 \pm 0,02$ & $0,6-1,0$ \\
$\mathrm{Mg}$ & $0,36 \pm 0,02$ & $0,36 \pm 0,01$ & $0,38 \pm 0,02$ & $0,34 \pm 0,01$ & $0,15-0,3$ \\
\hline
\end{tabular}

* Niveles óptimos de macronutrientes de acuerdo con Escobar et al. (2002).

ron síntomas de deficiencia y aun con la concentración de $200 \mathrm{mg} \mathrm{L}^{-1}$ se generan incrementos en crecimiento, en comparación con el tratamiento $150 \mathrm{mg} \mathrm{L}^{-1}$. Por lo tanto, todavía no se habría alcanzado el intervalo óptimo, de acuerdo a la relación que existe entre crecimiento y niveles de nutrientes en los tejidos de la planta (Landis 2000). Por otra parte, con los tratamientos $50 \mathrm{mg} \mathrm{L}^{-1} \mathrm{y}$ $100 \mathrm{mg} \mathrm{L}^{-1}$ las plantas tampoco alcanzaron el óptimo de nitrógeno foliar señalado por Escobar et al. (2002), lo que se presumía por los síntomas de deficiencia observados como un follaje pequeño y de color amarillo-verdoso (Landis 2000). Los restantes elementos (a excepción del fósforo para el tratamiento de $50 \mathrm{mg} \mathrm{L}^{-1}$ ) se encontraron en niveles adecuados, en todos los tratamientos, según los niveles óptimos propuestos por Escobar et al. (2002).

Los valores deficientes de nitrógeno foliar podrían ser explicados porque las concentraciones suministradas corresponden a aquellas comúnmente utilizadas en coníferas en el hemisferio norte (Landis 2000) y a que la frecuencia de aplicaciones fijas por semana no considera los cambios en la demanda hídrica del cultivo durante el ciclo de producción. Esta situación es posible mejorarla utilizando básicamente tres criterios: aplicando un esquema de fertirrigación en función de la demanda hídrica del cultivo, aumentando la concentración o implementando un sistema de fertilización exponencial (Timmer y Aidelbaum 1996), en donde la misma cantidad total de fertilizante usada por planta se agrega en concentraciones crecientes a lo largo del proceso de producción siguiendo la curva de crecimiento del cultivo y no un sistema convencional de concentración fija para toda la etapa de pleno crecimiento.

Las plantas tratadas con 150 y $200 \mathrm{mg} \mathrm{L}^{-1}$ deberían tener una mayor sobrevivencia y crecimiento como consecuencia de una mayor resistencia al estrés abiótico (Escobar y Sánchez 1992, Basaber 1993). Al respecto, Close et al. (2003) investigaron el comportamiento en terreno de plantas E. globulus originadas a partir de 10 diferentes viveros que producían plantas con amplias variaciones en atributos morfológicos y nutricionales. Encontraron que la media en crecimiento en altura de las plantas fue 35\% mayor en el mejor lote de plantas comparado con el peor, transcurridos seis meses después de realizada la plantación, y que el volumen del fuste difirió en un 50\% después de 18 meses. La altura de las plantas, el diámetro de cuello, el número de hojas, el desarrollo radical y las concentraciones foliares de fósforo, azufre, calcio, magnesio, sodio, cloro, fierro, manganeso, zinc, cobre y aluminio no explicaron las diferencias en crecimiento en altura postplantación; estas diferencias fueron correlacionadas con el volumen del contenedor o su profundidad y con la concentración foliar de nitrógeno. Concluyen que los mejores crecimientos en terreno se encontraron en plantas cuya concentración foliar de nitrógeno estaba entre 1,5 y 2,0\%.

Para finalizar, los resultados de las principales variables morfológicas y niveles foliares concuerdan con lo planteado por diversos autores, en que dosis crecientes de fertilización con macronutrientes, nitrógeno principalmente, generan mayores crecimientos en las plantas. Sin embargo, es importante seguir trabajando en caracterizar el efecto de todas las labores de vivero y en especial la fertilización sobre los distintos atributos que califican a una planta de calidad en vivero, y realizar los respectivos ensayos en terreno para poder obtener la retroalimentación necesaria para ajustar estas prácticas.

\section{CONCLUSIONES}

Para el potencial de crecimiento radical, las mejores respuestas se obtienen al fertilizar con 150 y $200 \mathrm{mg} \mathrm{L}^{-1}$. Los mayores atributos morfológicos se obtienen al fertilizar con 150 y $200 \mathrm{mg} \mathrm{L}^{-1}$ de nitrógeno.

Todas las concentraciones de nitrógeno suministradas al medio de crecimiento logran niveles deficientes de este elemento en el follaje. Lo mismo sucede para el fósforo a nivel foliar con el tratamiento de $50 \mathrm{mg} \mathrm{L}^{-1}$ de nitrógeno. El resto de los macroelementos alcanza niveles óptimos con todos los tratamientos suministrados.

A medida que aumenta la concentración de nitrógeno suministrada, aumenta el crecimiento en altura de las plantas, por lo tanto, el período de la fase de pleno crecimiento del cultivo disminuye. 


\section{AGRADECIMIENTOS}

Los autores agradecen al Vivero Forestal Proplantas Ltda. por su colaboración en la realización de este estudio. Al señor editor de la Revista Bosque, y a los señores árbitros por sus contribuciones en la publicación de este artículo.

\section{REFERENCIAS}

Arévalo J. 1994. Efecto del manejo de raíces y tallo en plantas de Eucalyptus globulus Labill. en vivero, en la resistencia a bajas temperaturas. Tesis Ingeniero Forestal. Concepción, Chile. Facultad de Ciencias Forestales. Universidad de Concepción. 56 p.

Basaber C. 1993. Efecto de diferentes calidades de plantas de las especies Eucalyptus globulus Labill., Pseudotsuga menziesii (Mirb.) Franco y Pinus radiata D. Don establecidas en distintas exposiciones y posiciones en la pendiente. Tesis Ingeniero Forestal. Concepción, Chile. Facultad de Ciencias Forestales, Universidad de Concepción. 135 p.

Burdett N. 1979. New methods for mensuring root growth capacity and their value in assessing Lodgepole pine stock quality. Can. J. For. Res. 9: 63-67.

Close DC, I Bail, CL Beadle, Q Clasen. 2003. Eucalyptus globulus Labill.: seedling specifications and performance after planting. Aust. For. 66: 145-152.

Close DC, I Bail, S Hunter, CL Beadle. 2005. Effects of exponential nutrient-loading on morphological and nitrogen characteristics and on after-planting performance of Eucalyptus globulus seedlings. For. Ecol. Man. 205: 397-403.

Cortina J, JL Peñuelas, J Puértolas, A Vilagrosa, R Savé. 2006. Calidad de planta forestal para la restauración en ambientes mediterráneos. Estado actual de conocimientos. Madrid, España. Organismo Autónomo Parques Nacionales, Ministerio de Medio Ambiente. 191 p.

Duryea ML, K McClain. 1984. Altering seedling physiology to improve reforestation success. In Duryea ML, GN Brown eds. Seedling physiology and reforestation manual. Success I. Proceedings of the Physiology Working Group Technical Session. Oregon State University. Corvallis, Oregon. USA. p. 77-114.

Escobar R, M Sánchez. 1992. Producción de Plantas Forestales: Algunos Aspectos. Boletín de Extensión No 51. Universidad de Concepción. Depto. Ciencias Forestales Chillán, Chile. $38 \mathrm{p}$.

Escobar R, M Sánchez, G Pereira. 2002. Forest nursery management in Chile. In Dumroese RK, LE Riley, TD Landis. National Proceedings: Forest and conservation nursery Associations. USDA Forest Service, Rocky Mountain Research Station. p. 219-225.

Fernández M, C Marcos, R Tapias, F Ruiz, F López. 2007. Nursery fertilisation affects the frost-tolerance and plant quality of Eucalyptus globulus Labill. Cuttings. Ann. For. Sci. 64: 865-873.

Grossnickle SC. 2000. Ecophysiology of Northern Spruce Species: The Performance of Planted Seedlings. Ottawa, Ontario, Canada, NRC Research Press 409 p.
Haase DL, R Rose (eds.). 1997. Symposium Proceedings: Forest Seedling Nutrition from the Nursery to the Field. Oregon State University, Nursery Technology Cooperative. $161 \mathrm{p}$.

Howell K, T Harrington. 2004. Nursery practices influence seedling morphology, field performance, and cost efficiency of containerized cherrybark oak. Southern Journal of Applied Forestry 28: 152-162.

Landis TD. 1985. Mineral nutrition as an index of seedling quality. En Duryea, M. (Ed): Evaluating seedling quality: principles, procedures and predictive abilities of mayor test. Forest Research Lab. Oregon State University Corvaillis. p. 29-48.

Landis TD. 2000. Manual de viveros para la producción de especies forestales en contenedor. In Landis TD, RW Tinus, SE McDonald, JP Barnett. Manual Agrícola. Volumen No 4. Washington, DC: U.S. Department of Agriculture, Forest Service. p. 1-67.

Little T, F Hills. 1978. Métodos Estadísticos para la Investigación en la Agricultura. México DF, México. Trillas. 271 p.

Malik V, VR Timmer. 1998. Biomass partitioning and nitrogen retranslocation in black spruce seedlings on competitive mixedwood sites: a bioassay study. Can. J. For. Res. 28: 206-215

Materán M, R Coopman, D Ríos, M Sánchez. 2004. Efecto del estrés nutricional de $\mathrm{N}$ sobre el crecimiento de plantas de Eucalyptus globulus Labill. Segundo Congreso Chileno de Ciencias Forestales. Valdivia. Chile.

Montgomery D. 1991. Diseño y Análisis de Experimentos. México DF, México. Grupo Editorial Iberoamérica. 590 p.

Navarro R, J Pemán. 1997. Apuntes de Producción de Planta Forestal. Córdoba, España. Ediciones Universidad de Córdoba. 267 p.

Novoa R, S Villaseca. 1989. Mapa Agroclimático de Chile. Santiago, Chile. Instituto de Investigaciones Agropecuarias. $221 \mathrm{p}$.

Peñuelas J, L Ocaña. 2000. Cultivo de Plantas Forestales en Contenedor. Madrid, España. Ediciones Mundi-Prensa. $190 \mathrm{p}$.

Salisbury F, C Ross. 2000. Fisiología Vegetal. Madrid, España. Thomson Editores, Spain Paraninfo. 988 p.

Shaw TM, JA Moore, JD Marshall. 1998. Root chemistry of Douglas-fir seedlings grown under different nitrogen and potassium regimes. Can. J. For. Res. 28: 1566-1573.

Timmer VR, AS Aidelbaum. 1996. Manual for exponential nutrient loading of seedlings to improve transplanting performance on competitive forest sites. Sault Ste Marie (Ontario): Canadian Forest Service, Natural Resources Canada. NODA/NFP Technical Report TR25. 21 p.

Van den Driessche R ed. 1991. Mineral nutrition of conifer seedlings. Boca Raton, FL, USA. CRC Press. 274 p.

Van den Driessche R. 1992. Changes in drought resistance and root growth capacity of container seedlings in response to nursery drought, nitrogen and potassium treatments. Can. J. For. Res. 22: 740-749.

Wang D, EP Bachelard, JC Banks. 1988. Growth and water relations of seedlings of two subspecies of Eucalyptus globulus. Tree Physiology 4: 129-138.

Recibido: 11.11 .08

Aceptado: 09.04.09 